\title{
Tendencias actuales, retos y oportunidades de los procesos de aprendizaje universitario aplicados a las Ciencias Forestales
}

\author{
Current Trends, Challenges and Opportunities of University Learning Processes Applied \\ to Forest Sciences
}

\section{Tendências atuais, desafios e oportunidades dos processos de aprendizagem universitária aplicados às ciências florestais}

\author{
Juan Carlos Valverde \\ Mariela Romero-Zúñiga ${ }^{2}$ \\ Lupita Vargas-Fonseca ${ }^{3}$
}

Recibido: marzo 2020

Aceptado: agosto 2020

Para citar este artículo: Valverde, J. C., Romero-Zúñiga, M., Vargas-Fonseca, L. (2020). Tendencias actuales, retos y oportunidades de los procesos de aprendizaje universitario aplicados a las Ciencias Forestales. Revista Científica, 39(3), 262-277. https://doi.org/10.14483/23448350.16030

\section{Resumen}

En las últimas décadas la introducción de nuevas tecnologías, técnicas de aprendizaje y métodos de enseñanza ha incidido en la simplificación del entendimiento de conceptos. La facilidad para encontrar información mediante fuentes de internet, redes sociales y medios interactivos ha generado el reforzamiento en los sistemas de aprendizaje dinámicos, en especial a nivel universitario. En el presente trabajo se estableció como objetivo analizar las tendencias de aprendizaje puestas en marcha en la educación superior, así como los retos y potenciales cambios que se deben considerar en próximos años con el fin de mejorar el aprendizaje y el incremento de conocimiento en estudiantes universitarios de Ciencias Forestales. Mediante una revisión bibliográfica se tuvieron en cuenta teorías psicológicas conductistas y cognitivas; además, se analizaron las tendencias de tipos de aprendizaje, desde la alternativa tradicional (memorística) hasta otras más nuevas como la cooperativa, emocional y latente, que estimulan el desarrollo y el uso de habilidades blandas. Los resultados evidenciaron que actualmente las tendencias se mueven hacia aprendizajes teórico-prácticos en los cuales la parte emocional y experimental toma relevancia y mejora la interacción con la realidad social y el entorno cercano. Finalmente, se consideraron las perspectivas a largo plazo en las que la implementación de e-learning (combinación de redes sociales), programas, videos y equipos informáticos impulsen el empoderamiento del nuevo conocimiento.

Palabras clave: aprendizaje, conocimiento, habilidades blandas, curva de aprendizaje, forestal.

1. Universidad de Concepción. Concepción, Chile. jcvalverde@itcr.ac.cr jcvalverde@outlook.com

2. Tecnológico de Costa Rica. Cartago, Costa Rica. mromero@tec.ac.cr

3. Tecnológico de Costa Rica. Cartago, Costa Rica. Ivargas@tec.ac.cr 


\section{Abstract}

In the last decades, the introduction of new technologies, learning techniques and teaching methods has had an impact on simplifying the understanding of concepts; the ease of finding information through Internet sources, social networks and interactive media has generated reinforcement in dynamic learning systems, especially at the university level. The objective of this work was to analyze the learning trends implemented at the university level, as well as the challenges and potential changes that should be considered in coming years, to improve learning and increase knowledge in science university students. Forestry. Employing a bibliographic review, behavioral and cognitive psychological theories were taken into account; Also, trends in types of learning were analyzed, from the traditional alternative (memory) to newer ones such as cooperative, emotional and latent, which stimulate the development and use of soft skills. The results showed that currently, the trends are moving towards theoretical-practical learning in which the emotional and experimental part takes relevance and improves the interaction with social reality and the intimate environment. Finally, the long-term perspectives were considered in which the implementation of e-learning (the combination of social networks), programs, videos, and computer equipment promote the empowerment of new knowledge.

Keywords: learning, knowledge, soft skills, learning curve, forestry.

\section{Resumo}

Nas últimas décadas, a introdução de novas tecnologias, técnicas de aprendizagem e métodos de ensino teve um impacto na simplificação da compreensão de conceitos; a facilidade de encontrar informações por meio de fontes da internet, redes sociais e mídias interativas tem gerado reforço em sistemas dinâmicos de aprendizagem, principalmente no nível universitário. O objetivo deste trabalho foi analisar as tendências de aprendizagem implementadas no nível universitário, bem como os desafios e potenciais mudanças que devem ser considerados nos próximos anos, a fim de meIhorar a aprendizagem e aumentar o conhecimento dos estudantes universitários de ciências. Silvicultura. Por meio de uma revisão bibliográfica, foram consideradas as teorias psicológicas comportamentais e cognitivas; Além disso, foram analisadas tendências nos tipos de aprendizagem, desde a alternativa tradicional (memória) até as mais novas como cooperativa, emocional e latente, que estimulam o desenvolvimento e o uso de soft skills. Os resultados evidenciaram que atualmente as tendências caminham para a aprendizagem teórico-prática em que a parte emocional e experimental ganha relevância e melhora a interação com a realidade social e o ambiente próximo. Por fim, foram consideradas as perspectivas de longo prazo em que a implantação de e-learning (combinação de redes sociais), programas, vídeos e equipamentos de informática promovem o empoderamento de novos conhecimentos.

Palavras-chaves: aprendizado, conhecimento, habilidades sociais, curva de aprendizado, silvicultura.

\section{Introducción}

El aprendizaje es el proceso en el que el ser humano adquiere conocimiento por medio del estudio o mediante nuevas experiencias que generan la modificación o adquisición de habilidades, destrezas o conductas (Hamidi y Chavosh, 2018; Hamidi y Jahanshaheefard, 2019). El aprendizaje se ha vinculado a procesos neurológicos como la memoria a corto y a largo plazo, en los cuales el individuo usa su capacidad de atención, razonamiento lógico o abstracto y distintas herramientas mentales para incorporar la información obtenida o mejorada dentro de sus capacidades de entendimiento del entorno (Zhang y Cui, 2018; Tsimane y Downing, 2020). Se ha determinado que aspectos como la motivación, el interés o la curiosidad por aprender incrementan las capacidades de memorización del conocimiento, y con ello se aumenta la retención de datos (Leal-Filho et al., 2018; Tvenge y Ogorodnyk, 2018); dicho aspecto es fundamental en el ser humano, sobre todo en edades adultas. Por lo anterior, el aprendizaje se presenta como un proceso individual, dinámico y constante (Badiaa y Chumpitaz-Campo, 2018; Tsimane y Downing, 2020). 
En las últimas décadas se ha desarrollado una gran variedad de teorías para el entendimiento del aprendizaje, así como de los factores de percepción positiva y negativa que infieren directa e indirectamente en el proceso de retención de conocimientos (Akkerman y Meijer, 2011). Aspectos como la etapa de crecimiento, la motivación personal, el entorno de desarrollo o la madurez emocional, entre otros (Carless, 2015; Muller y Tucker, 2017) pueden incidir en las capacidades cognitivas del individuo, en el empoderamiento de conocimiento y de estructuración de ideas, elemento fundamental para el razonamiento lógico y abstracto de la mente (Maclellan, 2004; Bailey y Garner, 2010; Monereo et al., 2013). Estudios de Hamidi y Chavoshi (2018) mencionan que es necesario el desarrollo de técnicas, metodologías y sistemas que proporcionen un mejoramiento de las cualidades integrales del individuo mediante el proceso de aprendizaje; con ello la estructuración de conocimiento será mejor y se incrementará la dinamización de nuevos conocimientos.

Un elemento pertinente por considerar en el proceso de aprendizaje es el grado de madurez emocional e intelectual del individuo (Leaman y Flanagan, 2013; Badia y Becerril, 2016). Nicol y MacfarlaneDick (2006) manifestaron que la motivación es un factor que determina el grado de éxito en la transferencia de conocimiento en ambientes universitarios. En su estudio señala que una parte del proceso de cambio del marco mental del estudiante consiste en el desarrollo de actividades, evaluaciones y correcciones en las que el estudiante se sienta animado, pueda empoderarse de conocimiento y rompa paradigmas sociales como el hecho de aprobar cursos para cumplir con un trámite de graduación. Por lo tanto, el elemento anímico intrínseco y extrínseco toma relevancia. Postareffa et al. (2012) destacan la necesidad de poner en funcionamiento sistemas motivacionales dentro de un esquema pedagógico que estimule al estudiante a incrementar constantemente su conocimiento y capacidades cognitivas.

Ante tal panorama se hace necesario disponer de disciplinas especializadas como la pedagogía, que se enfocan en la planificación, análisis y evaluación de aprendizaje, con el fin de crear técnicas $y$ herramientas que produzcan un impacto positivo en el estudiante (Carless, 2015). Las técnicas pedagógicas varían según la edad del estudiante; pues son distintos el sistema de aprendizaje de un niño que debe aprender conocimientos generales básicos y el de un adulto joven que cursa estudios universitarios. En ambos casos, el objetivo es que se dé una adopción de conocimientos y destrezas, pero el sistema de comunicación, interpretación y aplicación es diferente (DeCuir-Gunby et al., 2011; Davin et al., 2017). En la educación superior se debe tener claro que el proceso de aprendizaje, más que transmitir conocimiento, busca la creación de habilidades, destrezas y criterios necesarios para que el estudiante pueda desarrollar actividades laborales específicas que permitan solucionar problemas y hacer aportes a la sociedad (Hernández, 2012).

En concreto, en la región latinoamericana el proceso de aprendizaje en el ámbito universitario ha mostrado un constante cambio en las últimas tres décadas (Leaman y Flanagan, 2013). Tradicionalmente, se ha empleado el esquema de aprendizaje con un proceso de transmisión de conocimiento unidireccional y autoritario, en el cual tiene prevalencia la lección magistral; este ambiente ha creado un confort en el docente y una pérdida de interés por parte del estudiante (Monereo y Álvarez, 2013). Sin embargo, con los avances pedagógicos, el entendimiento de los procesos de asimilación y las nuevas teorías de aprendizaje, en la actualidad se usan sistemas dinámicos en los que docentes y estudiantes interactúan continuamente, se generen retroalimentaciones y empoderamiento de conocimiento.

De esta manera, se busca que el proceso sea continuo, se incremente la complejidad en el tiempo y se pueda generar un aprendizaje durable (Putnam y Borko, 2000). No obstante, las limitaciones presupuestarias y tecnológicas, en conjunto con el poco interés de cambio por parte de docentes y de algunas autoridades, inciden en que la posibilidad de innovación y mejora de procesos pedagógicos sea limitada (Warford, 2011). 
En el caso especial de las Ciencias Forestales, recientemente se ha dado un cambio como producto de la innovación en la instrumentación y en conocimientos que requieren aplicar modelos poco tradicionales, en los cuales la interacción entre estudiantes y docentes permita un empoderamiento y una asimilación concreta de la información (Agudo-Peregrina et al., 2014; Viberga et al., 2018). No obstante, la dinamización pedagógica, en conjunto con el desarrollo de nuevas tecnologías y conocimientos, en muchos casos infiere en los sistemas de aprendizaje puestos en marcha, los cuales tienden a quedar obsoletos en periodos cortos.

La FAO (2017) menciona que es necesaria, en primer lugar, la modificación de las técnicas de aprendizaje en forestería; en segundo lugar, la adecuación de las condiciones y los recursos disponibles en el sitio; y, en tercer lugar, el desarrollo de estrategias en las que los grupos meta asimilen e incorporen realmente la información. Según Vanclay (1996), este último aspecto es complicado, debido a que en muchos casos la formación académica del grupo meta es escasa, no se adapta al sistema de aprendizaje o requiere mayores periodos de aprendizaje.

Por lo anterior, el objetivo del presente trabajo consistió en realizar una recopilación de las tendencias actuales en torno a los procesos de aprendizaje universitario con potencialidad de aplicación en ciencias forestales, mediante el análisis de sus retos y oportunidades.

\section{Teorías psicológicas de aprendizaje universitario}

A nivel psicológico se han desarrollado tres líneas de entendimiento del proceso de aprendizaje (figura 1). En el caso de la formación universitaria, se tiene la preconcepción de que el estudiante cuenta con un grado de madurez mental elevado, posee conocimientos suficientes para la toma de decisiones y una estabilidad emocional suficiente para la formación profesional (Ali et al., 2013). En la actualidad, las tendencias psicológicas de aprendizaje son la conductista, cognitiva, conectiva y constructivista (Beck y Kosnik, 2006; Avella et al., 2016; Arnold y Sclater, 2017; Broos et al., 2018; Harfitta et al., 2018).

La teoría conductista parte del concepto de que el aprendizaje es producto del acondicionamiento de los estímulos a los que se expone el estudiante; en el proceso, la persona interactúa e incluye en su conducta la información adquirida (Boyle-Baise y Sleeter, 1998; Akkerman y Meijer, 2011), de forma tal que un estímulo positivo refuerza el aprendizaje y genera un crecimiento en la capacidad de entendimiento del estudiante; por otro lado, el estímulo negativo lo excluye y lo inhibe en el entendimiento y en la asimilación de información (Viberga et al., 2018).

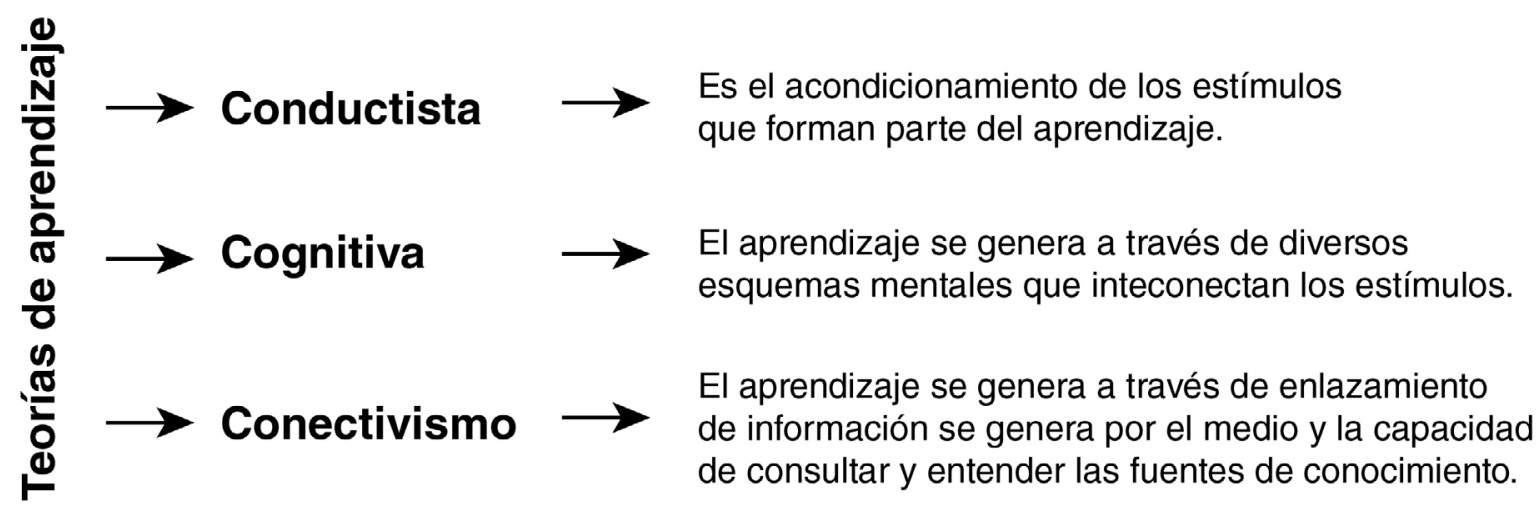

Figura 1. Teorías de aprendizaje actuales en la enseñanza universitaria.

Fuente: basado en Boyle-Baise y Sleeter (1998); Akkerman y Meijer (2011). 
La teoría cognitiva plantea que el aprendizaje se obtiene mediante diversos esquemas mentales en los que se da una asociación de términos y conceptos para que el estudiante pueda generar ideas claras de un término, proceso o habilidad (Hernández, 2012). Conforme mayores sean las habilidades blandas del usuario y su capacidad de adaptación al conocimiento transmitido, el aprendizaje se mejorará y la capacidad de absorción de conocimiento se incrementará (Monereo et al., 2013). Sin embargo, cuanto menor sea el dinamismo o el interés de la persona, las conexiones mentales serán menores y no se tendrá la misma retención (Tsimane y Downing, 2020).

La teoría de conectivismo es de las más recientes en pedagogía; se sustenta bajo la concepción de que el estudiante puede incrementar su conocimiento y habilidades con la combinación de la información disponible en el ambiente, en conjunto con las habilidades conectivas que le permiten realizar un análisis crítico (Agudo-Peregrino et al., 2014). En este proceso se combinan ambas fuentes de información, lo cual permite que el usuario maximice su entendimiento y pueda complementar los conocimientos que posee.

Finalmente, la teoría constructivista se enfoca en el alumno como un ser responsable que participa activamente en su proceso de aprendizaje, por lo que sus aportes dentro y fuera de clase, en el desarrollo de la práctica y del trabajo, en conjunto con la realización activa de consultas, genera una gran relevancia en el proceso de aprendizaje activo (Agudo-Peregrino et al., 2014).

En el ámbito universitario, las tendencias actuales son conductistas y conectistas, debido a que se apuesta a un aprendizaje integral en que el estudiante pueda disponer de conocimientos suficientes que le permitan responder con criterio profesional a las situaciones que enfrente, al combinar la información disponible en plataformas físicas y digitales (Brayko, 2013). Múltiples autores destacan que los avances tecnológicos y la incorporación de nuevos programas y equipos informáticos inciden para que el aprendizaje se dinamice, al dejar en evidencia que los sistemas tradicionales dejan ser funcionales y se vuelven obsoletos (Coffey, 2010; Gross y Rutland, 2017). Por lo anterior, se debe iniciar una incorporación y dinamización del aprendizaje, de manera tal que el usuario combine los sistemas tradicionales con estas nuevas herramientas para así mejorar su capacidad de aprendizaje y criterio profesional.

\section{Tendencias actuales de los tipos de aprendizaje}

Múltiples estudios pedagógicos (Zeichner, 2010; Csikosova et al., 2012; Šolc, et al., 2012, Kovacova y Vackova, 2015; Willamo et al., 2018; Guzmán et al., 2019) enfocados a nivel universitario destacan ocho tipos de aprendizaje (figura 2). Las diferencias entre cada uno se dan como producto de la parametrización de las habilidades y las capacidades de aprendizaje del estudiante, así como por la complejidad del conocimiento impartido y el tiempo dado para el aprendizaje. Según dichas características, el proceso puede ser funcional y positivo para el usuario e incrementar sus habilidades; por el contrario, también puede generar frustración o desinterés. Los tipos de aprendizaje identificados fueron: receptivo, descubrimiento, memorístico, significativo, observacional, latente, emocional y cooperativo.

El aprendizaje receptivo es un proceso dinámico en el que el estudiante primero se expone al conocimiento al aprender y comprender lo nuevo y luego desarrolla habilidades de análisis y aplicación de ello. Este sistema no representa un descubrimiento de conocimiento para el estudiante, debido a que la información se proporciona directamente (Zeichner, 2010); la desventaja es que, si tiene dificultad para entender conceptos de forma indirecta, generará una limitación al interactuar con ese conocimiento (Ryan, 2011). Por otro lado, en el sistema de aprendizaje por descubrimiento el estudiante emprende por iniciativa propia y progresiva la localización y asimilación de conocimientos, desde una perspectiva de reto. A 


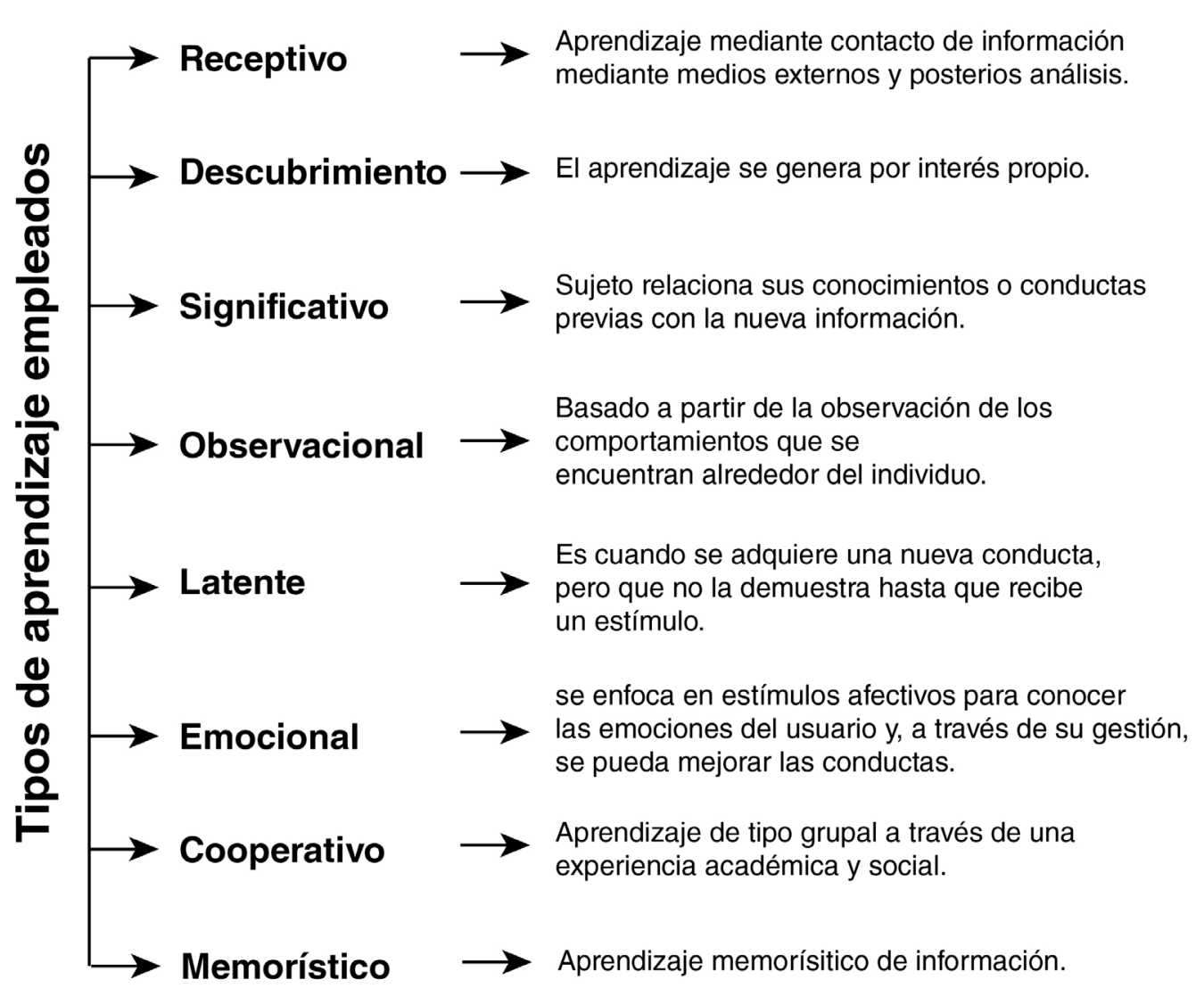

Figura 2. Tipos de aprendizaje implementados en la enseñanza universitaria. Fuente: basado en Boyle-Baise y Sleeter (1998); Akkerman y Meijer (2011).

nivel universitario es una excelente estrategia para aprendizaje de temas teóricos o de vanguardia (Gross y Rutland, 2017); la principal limitante es que depende de las habilidades del estudiante y de la complejidad del tema (Postareffa et al., 2012).

El aprendizaje significativo funciona a partir de la relación de los saberes propios con el conocimiento adquirido en lecciones, de forma tal que se genere una asimilación mediante una restructuración del conocimiento de manera coherente. Este tipo de aprendizaje es altamente funcional en temáticas artísticas y deportivas (Ali et al., 2013). Sin embargo, tiene la desventaja de que depende de la velocidad de adaptación del conocimiento por parte del estudiante, así como del grado de modificación que se requiera (Harfitta et al., 2018).

Por su parte, el sistema observacional se basa en la capacidad de observación del estudiante en relación con su entorno y, por lo tanto, su tendencia a repetir actividades. Este es común en aprendizaje de arte o técnicas básicas de laboratorio; es muy intuitivo, pero limitado al ambiente, y las actividades que se desarrollan se dan de una manera coherente (Ryan, 2011). En el sistema latente, un usuario adquiere una nueva conducta, pero no la demuestra hasta que recibe un estímulo para ello. En la educación superior es poco usado, debido a su complejidad y a que depende de la capacidad de respuesta del estudiante (Zhang y Cui, 2018). El sistema de aprendizaje emocional se enfoca en estímulos afectivos para conocer las emociones del sujeto por medio de su gestión y mejorar las conductas en las personas. Es un aprendizaje que aporta beneficios psicológicos y personales, lo cual genera un estado anímico positivo. Su limitación es que depende de las capacidades afectivas 
y emocionales del docente, así como del tipo de tema que se desarrolle (Hernández, 2012; Kovacova y Vackova, 2015).

El sistema cooperativo es óptimo para el trabajo en equipo. Consiste en la interacción del alumno con otras personas, lo cual permite aprender mediante experiencias académicas y sociales. La dificultad de este sistema es que depende de la conformación de los grupos de trabajo y el grado de interés en el tema (Gross y Rutland, 2017).

Finalmente, el memorístico es un sistema tradicional en que el estudiante debe asimilar el conocimiento y repetirlo constantemente, con el fin de aprender de memoria la información. Este sistema es útil para conocimientos teóricos o abstractos, pero cuenta con limitaciones en cuanto a retención y aprendizaje (Gross y Rutland, 2017). En Ciencias Forestales se debe contar con una evaluación previa del tipo de destrezas con las que cuenta el grupo meta, al identificar sus fortalezas y debilidades, y con ello desarrollar una estrategia de transferencia de conocimientos que explote al máximo dicha condición. Con ello el proceso será simple, optimizará las cualidades del grupo y disminuirá los errores de aprendizaje.

\section{Modelo de aprendizaje universitario}

Cuando se menciona un modelo de aprendizaje los elementos que lo conforman han variado significativamente en la última década. En el pasado se limitaba a aspectos de contenido del conocimiento y a la técnica de transmisión; con lo cual se daba, en muchos casos, un abordaje superficial del usuario meta o su total desconsideración (Dean y Levis, 2016). Sin embargo, los avances en pedagogía y neurociencia abordan elementos nuevos que tienden a centrarse más en este.

En la figura 3 se presenta un concepto de aprendizaje que va desde los microelementos a macroelementos. Primeramente, en el aspecto del estudiante se deben analizar sus expectativas y concepciones previas al tema. Mork et al. (2016) destacan que las expectativas de un estudiante son clave para el éxito o fracaso de un plan de aprendizaje; una percepción positiva del estudiante influye en que el conocimiento sea asimilado de buena manera, motiva al estudiante a expandir sus habilidades, a que aprenda nuevos conocimientos de forma propia y que sienta el proceso como un juego, más que como un elemento pesado o condicionado a una nota o cumplimiento de un requisito.

Complementario a las expectativas se encuentra la adaptación de las circunstancias, otro elemento intrínseco; conforme mejores sean el ambiente, la motivación y la libertad de aprendizaje con que cuente el estudiante, mayor adaptabilidad y capacidad de respuesta tendrá. La motivación de aprendizaje incide directamente en la capacidad receptiva del conocimiento y la habilidad de respuesta a situaciones en las que el conocimiento debe aplicarse (Dlouhá et al., 2013). Finalmente, el aspecto de conocimientos previos cuenta con un peso considerable al momento de las acciones y respuestas. Rieckmann (2012) destaca que el conocimiento previo puede ser implementado para el desarrollo de estrategias de respuesta a condiciones dadas o para asimilar un nuevo conocimiento; es un parámetro que permite al estudiante entender las condiciones que se expone y tomar decisiones. Conforme mayor sea la experiencia, menor será la cautela al momento de exponerse a un nuevo conocimiento. Tsimane y Downing (2020) resaltan que uno de los aspectos positivos que tiene la experiencia previa es la capacidad de incrementar el tiempo y la motivación para un nuevo conocimiento, pero con la limitante de que en temas con experiencias previas negativas se condiciona a resultados que tienden a ser deficientes. En relación con aspectos externos se destacan dos: influencias sociales y el esquema del sistema (figura 3). En los últimos años, la influencia social ha tenido un aumento de relevancia debido al extendido uso de redes sociales como medios de comunicación, información y contacto de las nuevas generaciones (Hosseini, 2011). 


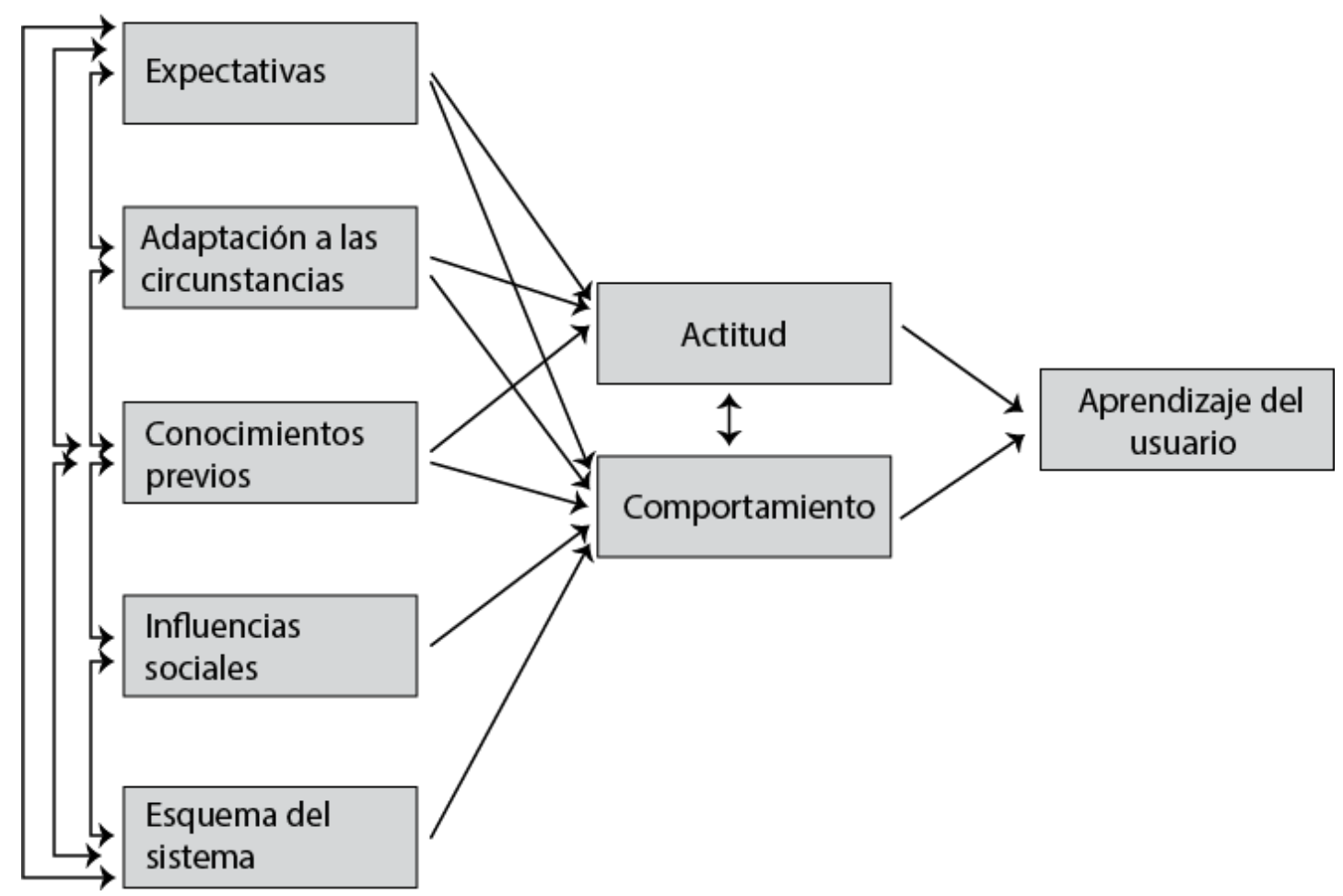

Figura 3. Modelo teórico de simulación de aprendizaje implementado para estudiantes universitarios.

Fuente: basado en Boyle-Baise y Sleeter (1998); Akkerman y Meijer (2011).

La utilización de nuevos medios implica que los sistemas de aprendizaje cambien. La exploración y la adaptación a medios digitales que informan en segundos sobre cualquier temática de relevancia buscan que el usuario considere simple adquirir el conocimiento; dicho aspecto se está empezando a considerar en la educación (Braithwaite y Corr, 2016). La implementación de canales interactivos, videos explicativos de la limitación de los sistemas y la simplificación de la distancia de comunicación entre docentes y estudiantes incide favorablemente en el aprendizaje (Gorgan, 2015).

Es importante destacar que las redes sociales también pueden tener un papel negativo. Por ejemplo, la creación de percepciones negativas hacia un curso, programa o tema en específico; la generación de información falsa o mala comunicación, lo que puede provocar confusión en el docente; finalmente, la simplificación de la realidad, que genera dependencia de los medios sociales como mecanismo de información (Klement, 2015).

El esquema del sistema es uno de los elementos de mayor peso en el proceso de aprendizaje, pero en muchos casos es considerado un elemento estático, rígido, que no se adapta a la realidad de los temas de aprendizaje o el estudiante (Manța et al., 2015). Los esquemas, por lo general, consideran tanto el sistema de enseñanza, evaluación y metas del curso o proceso de aprendizaje; pero se suele ignorar el conocimiento previo y las habilidades del estudiante, lo que en muchos casos produce que el proceso sea complicado o fallido (Hernández, 2012; Gabdulchakov, 2014; Arnold y Sclater, 2017).

\section{Aprendizaje en Ciencias Forestales}

Cada formación profesional cuenta con elementos diferenciadores únicos en el proceso de enseñanza 
(tabla 1). Estos se deben adaptar, considerar y optimizar con el fin de que el aprendizaje sea eficaz y se genere la mejor experiencia posible para el estudiante. Tradicionalmente, los modelos de aprendizaje puestos en funcionamiento se basan en el cognitivo-memorístico (Zeichner, 2010; Šolc et al., 2012; Kovacova y Vackova, 2015). La relación entre teoría y práctica es fundamental para la correcta asimilación de conocimiento y para el incremento de habilidades. Ameyaw et al. (2016) mencionan que el desarrollo de modelos mixtos en los que los conceptos teóricos se combinen con la práctica mejora el entendimiento del tema, permite generar y aclarar dudas, además de que con el tiempo permite dar un concepto claro y entendible del impacto de conocer teoría al momento de solucionar problemas. En Ciencias Forestales tal aspecto es un componente fundamental en el entendimiento y la gestión de recursos arbóreos y ecosistemas.

El elemento de desarrollo de giras de campo es un aspecto relacionado con la variable anterior. Cuanto mayor realismo y contexto tenga el caso de estudio, será mejor el entendimiento y la aplicación de nuevos conocimientos. Leal-Filho et al. (2018) destacan que el proceso de aprendizaje puede tener elementos de estímulo para el estudiante; tales como prácticas reales de casos en los que se aplique conocimiento, o el cambio de las condiciones de estudio, en conjunto con la participación de instructores parte al docente. Esto permite incrementar la motivación, el deseo de aprendizaje y la retroalimentación de la evaluación; de tal manera, hay una mejora en las habilidades del estudiante en cuanto a entendimiento y realización de acciones, con la intención de generar un producto o resultado.

Sin embargo, con respecto al trabajo en grupo y al desarrollo de habilidades blandas, en la mayoría de las formaciones profesionales se exige el trabajo con comunidades que poseen distintos niveles de educación y participación en distintos grupos interdisciplinarios, lo cual tiene una exigencia mayor (Willamo et al., 2018). Por lo general, la formación académica se enfoca en conocimientos y elementos teóricos, pero se colocan en un segundo plano las habilidades de interacción en grupos interdisciplinarios y el desarrollo de habilidades que mejoren el comportamiento y la capacidad de respuesta del estudiante. Hernández (2012) menciona que las tendencias actuales buscan una formación integral del profesional, para que sus habilidades técnicas no sean el único enfoque y más bien mejore su capacidad de interacción y atención con el entorno. Según Rieckmann (2012), tal aspecto es fundamental para el desarrollo de trabajos multidisciplinarios y programas de transferencia de conocimientos.

Finalmente, el aspecto de trabajo con comunidades es un elemento intrínseco y de relevancia en la formación en Ciencias Forestales (Akkerman y Meijer, 2011). Las tendencias de crecimiento en habilidades blandas, en conjunto con la práctica de conocimientos, son fundamentales para el desarrollo de modelos de docencia en que el estudiante pueda interactuar, participar y transferir el conocimiento adquirido a comunidades o grupos meta.

\section{El papel de la motivación interna y externa}

Un elemento que se debe considerar en los procesos de aprendizaje es el papel de la motivación en la capacidad de impulsar habilidades en el estudiante o de inhibir el interés por aprender. Avella et al. (2016) destacan la motivación como un elemento que preinscribe al estudiante en su entendimiento de los nuevos conocimientos; conforme mayor sea el grado de seguridad y confianza ante la nueva experiencia, se podrá incrementar la satisfacción en el entendimiento del tema.

Por su parte, Carless (2015) considera que la generación de ambientes universitarios en los que el estudiante se encuentre en confianza permite que la percepción de temor al nuevo contenido de información sea baja; con ello se incrementa la posibilidad de éxito en el aprendizaje. La relación entre un ambiente externo que inspire confianza y una percepción interna positiva hacia el nuevo 
Tendencias actuales, retos y oportunidades de los procesos de aprendizaje universitario aplicados a las Ciencias Forestales

Valverde, J. C., Romero-Zúñiga, M., Vargas-Fonseca, L.

Tabla 1. Variables diferenciadoras relevantes en estudiantes universitarios en Ciencias Forestales

\begin{tabular}{|c|c|c|c|}
\hline Variable & $\begin{array}{l}\text { Relevancia en el } \\
\text { aprendizaje }\end{array}$ & $\begin{array}{l}\text { Limitación con sistemas } \\
\text { actuales }\end{array}$ & $\begin{array}{l}\text { Perspectivas para el } \\
\text { futuro }\end{array}$ \\
\hline $\begin{array}{l}\text { Relación teoría-práctica } \\
\text { de conocimientos. }\end{array}$ & $\begin{array}{l}\text { Los futuros profesionales } \\
\text { en Ciencias Forestales } \\
\text { no solo deben conocer } \\
\text { terminologías teóricas en su } \\
\text { conocimiento, sino también } \\
\text { poderlas aplicar y explicar } \\
\text { en la actividad laboral. }\end{array}$ & $\begin{array}{l}\text { No todas las temáticas } \\
\text { se adaptan a un sistema } \\
\text { teórico-práctico. }\end{array}$ & $\begin{array}{l}\text { Estructurar cursos en los } \\
\text { que la parte práctica y la } \\
\text { aplicación de casos sean } \\
\text { amplias y permitan al } \\
\text { futuro egresado tener un } \\
\text { mayor conocimiento. }\end{array}$ \\
\hline $\begin{array}{l}\text { Desarrollo de giras de } \\
\text { campo. }\end{array}$ & $\begin{array}{l}\text { Son fundamentales para } \\
\text { que el estudiante pueda } \\
\text { aplicar su conocimiento } \\
\text { en casos reales y así } \\
\text { conocer la realidad } \\
\text { nacional. }\end{array}$ & $\begin{array}{l}\text { La lejanía de los sitios de } \\
\text { estudio y el sitio de caso } \\
\text { limita la exposición a } \\
\text { situaciones reales para los } \\
\text { estudiantes. }\end{array}$ & $\begin{array}{c}\text { Desarrollo de trabajos } \\
\text { que busquen la solución } \\
\text { de casos reales. }\end{array}$ \\
\hline $\begin{array}{l}\text { Trabajo en grupo } \\
\text { y desarrollo de } \\
\text { habilidades blandas. }\end{array}$ & $\begin{array}{c}\text { Es fundamental que } \\
\text { el egresado pueda } \\
\text { incrementar habilidades } \\
\text { para trabajos en } \\
\text { comunidades y con otros } \\
\text { profesionales }\end{array}$ & $\begin{array}{c}\text { Los sistemas tradicionales } \\
\text { no desarrollan de } \\
\text { forma apropiada dichas } \\
\text { habilidades. }\end{array}$ & $\begin{array}{c}\text { Se deben proponer } \\
\text { trabajos en los que } \\
\text { el estudiante pueda } \\
\text { incrementar habilidades } \\
\text { blandas y crecimiento } \\
\text { personal. }\end{array}$ \\
\hline $\begin{array}{l}\text { Trabajo con } \\
\text { comunidades. }\end{array}$ & $\begin{array}{l}\text { Es pertinente que el futuro } \\
\text { profesional cuente con } \\
\text { las habilidades suficientes } \\
\text { para el trabajo en equipo. }\end{array}$ & $\begin{array}{l}\text { Los sistemas tradicionales } \\
\text { no desarrollan de } \\
\text { forma apropiada dichas } \\
\text { habilidades. }\end{array}$ & $\begin{array}{l}\text { Se debe crear conciencia } \\
\text { y canales de trabajo en } \\
\text { los que los estudiantes } \\
\text { puedan trabajar con } \\
\text { casos reales. }\end{array}$ \\
\hline
\end{tabular}

Fuente: basado en Zeichner (2010); Šolc et al. (2012); Kovacova y Vackova (2015).

aprendizaje incide en que el proceso sea adecuado y mejoren en el tiempo la capacidad y la experiencia de la persona a su nuevo conocimiento.

Por lo tanto, en temáticas que combinen relación de conceptos teóricos con prácticos y con temas de realidad social, el abordaje de transferencia de conocimiento debe considerar los tres elementos dados por Gorgan (2015) y Klement (2015):

i. Creación de canales de comunicación continuos en los que el estudiante cuente con un acompañamiento y con espacios de comunicación, aclaración de dudas y expresión de su sentir: esto tiene como fin mejorar la relación con el cuerpo docente y simplificar su temor a fallar.

ii. El empoderamiento de conocimiento es fundamental, por lo que disponer de periodos en los que el usuario pueda aumentar su conocimiento y experiencia mejoran su motivación y ánimo hacia el nuevo tema, habilidad o conocimiento a incorporar.

iii. Los procesos continuos de aprendizaje, el desarrollo del curso y las lecciones deben permitir un constante crecimiento intelectual por parte del estudiante.

\section{Limitaciones de los sistemas tradicionales de aprendizaje}

En muchos casos, los sistemas tradicionales de enseñanza se limitan al autoritarismo del docente y a desarrollar clases unidireccionales de información en formato de clases magistrales (Ali et al., 2013). Esto incide en que el aspecto motivacional disminuya y se limite a la obtención de una nota 
o al cumplimiento de un estándar mínimo de calidad solicitado por el sistema, lo cual provoca que la capacidad de retención de conocimiento sea mínima (Arnold y Sclater, 2017). Aspecto que incide en que la calidad de conocimiento profesional sea mayor y el fracaso del proceso tienda a ser mínimo.

Otra limitación del sistema tradicional es su incapacidad para adaptarse e incorporar los avances tecnológicos. De forma general, los asumen como un mecanismo de referencia o de obtención de información para presentación de informes o trabajos (Dean y Levis, 2016), pero no se les da un papel relevante para el desarrollo de modelos mixtos y creativos en los que el trabajo en grupo, el hallazgo de información y el aplacamiento de las nuevas teorías de aprendizaje incidan positivamente en el proceso.

\section{Tendencias de aprendizaje universitario}

En la última década las tendencias en educación universitaria se han enfocado en la implementación del e-learning, lo cual implica el empleo de dispositivos móviles, tabletas o computadoras; pues, mediante programas, aplicaciones o juegos se transfiere el conocimiento a los estudiantes (Gabdulchakov, 2014). Múltiples estudios en universidades de todo el mundo han mostrado los efectos positivos y negativos que tiene la implementación de ambientes digitales en el proceso de enseñanza universitaria. Entre los aspectos positivos, Hosseini (2011) menciona que la competitividad de aprendizajes es un estilo de vida en la actualidad; los usuarios tienden a usar dispositivos electrónicos para atender una amplia gama de necesidades, por ejemplo, aspectos de comunicación, socialización, cálculo, control de actividades, entre otros.

La implementación de plataformas digitales como juegos o videos, en conjunto con la dinamización y utilización del tiempo por parte del estudiante, incrementa el éxito del proceso. Por ejemplo, Dean y Levis (2016) determinaron que hay una mejora en el rendimiento académico en estudiantes de Ciencias de la Salud tras la implementación de videos cortos explicativos de terminología y conceptos que estudiantes avanzados tienden a no dominar o comprender con claridad; con ello se mejoró en un 30 \% la capacidad de entendimiento y asimilación del conocimiento.

Por su parte, Zeichner (2010) determinó en estudiantes universitarios que el uso de programas de diseño combinado con actividades participativas mejoró la capacidad creativa del grupo de estudio, sobre todo en cuanto a conceptos de distribución de espacio y manejo de la luz. Asimismo, Carless (2015) resalta que se deben desarrollar planes didácticos en los que se ponga en funcionamiento la herramienta digital como un complemento y no como el elemento fundamental para el éxito del proceso de aprendizaje. En el área forestal la implementación de programas en las áreas de sistemas de información geográfica, estadística o economía ha ganado importancia en los últimos años debido a su facilidad de sistematización de los procedimientos, simplificación en el entendimiento sobre cómo desarrollar las actividades y la visualización de los resultados con prácticas reales.

La implementación de aprendizajes con sistemas electrónicos como videos, clases pregrabadas o videos interactivos mejora la experiencia de aprendizaje, disminuye el tiempo de entendimiento y permite al estudiante utilizar el material disponible según sus necesidades. Csikosova et al. (2012) destacan que el uso de videos en línea simplifica el entendimiento de conceptos teóricos, ya que la utilización de estos, de imágenes o cortos animados mejora el entendimiento de los conceptos y la manera en que se pueden aplicar en la solución de problemas reales. Por su parte, MacleIlan (2004) menciona que la implementación de medios electrónicos permite al estudiante disponer de información que en el momento considere necesario, así como adaptar el aprendizaje a su ritmo y repasar la cantidad de veces que desee.

Son múltiples los estudios que determinan que el futuro de la enseñanza se centrará en la 
utilización de medios tecnológicos, como simulaciones 3D, realidad virtual, videos interactivos y aplicaciones portables de uso acorde a la necesidad del estudiante (Warford, 2011).

No obstante, en las Ciencias Forestales siempre se ha presentado una tendencia conservadora hacia la utilización de nuevas tecnologías. Esto incide en que los procesos de aprendizaje sean lentos y en muchos casos no se adquiera todo el conocimiento necesario o se adapte a las necesidades de usuarios cambiantes, cada vez más tecnológicos y con menos disposición de tiempo para métodos tradicionales (Klement, 2015). Asimismo, Davin et al. (2017) mencionan que en el futuro la utilización de cursos virtuales, tanto sincrónicos como asincrónicos, crecerá y por ello es necesario que las Ciencias Naturales emigren a nuevas metodologías.

\section{Conclusiones}

Se deben analizar y restructurar los esquemas de aprendizaje actuales. Por lo general, se han utilizado modelos estáticos conductistas que evitan una adaptación del sistema de aprendizaje a las cualidades, limitaciones y necesidades del estudiante. En la actualidad se dispone de nuevas teorías de aprendizaje como la conductista, conectivista y constructivista, las cuales proponer mejorar el desempeño del estudiante sin generar afectaciones emocionales. Además, se analizaron las tendencias actuales sobre tipos de aprendizaje, específicamente las ocho técnicas más estudiadas, desde la alternativa tradicional (memorística) hasta las nuevas tendencias como la cooperativa, emocional y latente que estimulan el desarrollo y uso de habilidades blandas, así como el mejoramiento de la interacción con la realidad social y el entorno cercano. El comportamiento actual se encamina hacia el desarrollo de técnicas que combinen la motivación, el conocimiento previo y los valores con los cuales cuenta el estudiante.

Además, se determinó como pertinente el cambio del sistema de aprendizaje universitario al pasar de un modelo estructurado, con un sistema de temas y objetivos definidos en los que el estudiante tenía que adaptarse, a uno en el que es la prioridad. Así, se desarrolla contenido de forma que el aprendizaje sea el máximo objetivo, y en el que aspectos emocionales, experiencias previas y habilidades blandas incidan en una mejora del rendimiento académico. Hoy día el aprendizaje es producto de una interacción de múltiples variables que pueden afectar positiva o negativamente al estudiante.

Finalmente, se consideraron las perspectivas a largo plazo en las que la implementación de e-learning impulse el empoderamiento de nuevos saberes, la digitalización de cursos, implementación de videos, animaciones, modelos 3D y otros recursos tecnológicos. En esta nueva tendencia el estudiante podrá aprender a su ritmo, con capacidad ilimitada para repasar los conceptos que considere necesarios y con una amplia variedad de sistemas tecnológicos que le permitirán adaptarse a su gusto. El cambio de un esquema rígido a uno flexible ha de ganar importancia en los próximos años.

\section{Referencias}

Agudo-Peregrina, A., Iglesias-Pradas, S., Conde-González, M., Hernández-García, A. (2014). Can we predict success from log data in VLEs? Classification of interactions for learning analytics and their relation with performance in VLE-supported F2F and online learning. Computers in Human Behavior, 31, 542-550. https://doi.org/10.1016/j.chb.2013.05.031

Akkerman, S., Meijer, P. (2011). A dialogical approach to conceptualizing teacher identity. Teaching and Teacher Education, 27, 308-319. https://doi.org/10.1016/j.tate.2010.08.013

Ali, M., Asadi, M., Gašević, J., Jovanovic, M., Hatala, M. (2013). Factors influencing beliefs for adoption of a learning analytic tool: An empirical study. Computers y Education, 62, 130-148. https://doi.org/10.1016/j. compedu.2012.10.023 
Ameyaw, B., Arts, B., Wals, A. (2016). Challenges to responsible forest governance in Ghana and its implications for professional education. Forest Policy and Economics, 62, 78-87. https:// doi.org/10.1016/j.forpol.2015.07.011

Arnold, K., Sclater, N. (2017). Student perceptions of their privacy in learning analytics applications. Proceedings of the seventh international learning analytics y knowledge conference, ACM, 66-69. https://doi. org/10.1145/3027385.3027392

Avella, J., Kebritchi, M., Nunn, S., Kanai, T. (2016). Learning analytics methods, benefits, and challenges in higher education: A systematic literature review. Online Learning, 20, 13-29. https://doi.org/10.24059/olj.v20i2.790

Badia, A., Becerril, L. (2016). Renaming teaching practice through teacher reflection using critical incidents on a virtual training course. Journal of Education for Teaching, 42, 224-238. https:// doi.org/10.1080/02607476.2016.1143146

Badiaa, A., Chumpitaz-Campo, L. (2018). Teachers learn about student learning assessment through a teachereducation process. Studies in Educational Evaluation, 58, 1-8. https://doi.org/10.1016/j.stueduc.2018.05.004

Bailey, R., Garner, M. (2010). Is the feedback in higher education assessment worth the paper it is written on? Teachers' reflections on their practices. Teaching in Higher Education, 15, 187-198. https://doi.org/10.1080/13562511003620019

Beck, C., Kosnik, C. (2006). Innovations in teacher education: A social constructivist approach. The Suny Press, New York , 1-6.

Boyle-Baise, M., Sleeter, C. (1998). Community service learning for multicultural teacher education. Education Resources Information Center, Washington, DC , 1-5.

Braithwaite, R., Corr, P. (2016). Hans Eysenck, education and the experimental approach: A meta-analysis of academic capabilities in university students. Personality and Individual Differences, 103, 163-171. https://doi.org/10.1016/j.paid.2016.03.040
Brayko, K. (2013). Community-based placements as contexts for disciplinary learning: A study of literacy teacher education outside of school. Journal of Teacher Education, 64, 47-59. https://doi.org/10.1177/0022487112458800

Broos, T., Verbert, K., Langie, G., Van Soom, C., De Laet, T. (2018). Multi-institutional positioning test feedback dashboard for aspiring students: Lessons learnt from a case study in flanders. Proceedings of the $8^{\text {th }}$ international conference on learning analytics and knowledge, ACM, 51 55. https://doi.org/10.1145/3170358.3170419

Carless, D. (2015). Exploring learning-oriented assessment processes. Higher Education, 69, 963-976. https://doi.org/10.1007/ s10734-014-9816-z

Coffey, H. (2010). "They taught me": The benefits of early community-based field experiences in teacher education. Teaching and Teacher Education, 26, 335-342. https://doi.org/10.1016/j. tate.2009.09.014

Csikosova, A., Teplicka, K., Senova, A. (2012). Communication and Humanization of University Education Through E-Learning. Procedia - Social and Behavioral Sciences, 46, 2978-2982. https://doi.org/10.1016/j.sbspro.2012.05.600

Davin, K., Herazo, J., Sagre, A. (2017). Learning to mediate: Teacher appropriation of dynamic assessment. Language Teaching Research, 21(5), 632-651. https:// doi.org/10.1177/1362168816654309

Dean, M., Levis, A. (2016). Does the use of a university lecturer as a visiting tutor support learning and assessment during physiotherapy students' clinical placements? A survey of higher education institution providers. Physiotherapy, 102, 365-370. https://doi.org/10.1016/j. physio.2015.08.010

DeCuir-Gunby, J., Marshall, P., McCulloch, A. (2011). Developing and using a codebook for the analysis of interview data: An example from a professional development research project. Field Methods, 23(2), 136-155. https:// doi.org/10.1177/1525822X10388468 
Dlouhá, J., Huisingh, D., Barton, A. (2013). Learning networks in higher education: universities in search of making effective regional impacts. Journal of Cleaner Production, 49, 5-10. https://doi.org/10.1016/j.jclepro.2013.01.034

FAO. (2007). Learning for the future: forestry training and education. Rome, Italy. 13p.

Gabdulchakov, V. (2014). Personification of Multicultural Education in the Universities of Russia (Analysis of Training Specialists for Kindergartens). Procedia - Social and Behavioral Sciences, 146, 129-133. https://doi.org/10.1016/j. sbspro.2014.08.099

Gorgan, V. (2015). Requirement Analysis for a Higher Education Decision Support System. Evidence from a Romanian University. Procedia - Social and Behavioral Sciences, 197, 450-455. https://doi.org/10.1016/j.sbspro.2015.07.165

Gross, Z., Rutland, S. (2017). Experiential learning in informal educational settings. International Review of Education, 63, 1-8. https://doi. org/10.1007/s11159-017-9625-6

Guzman, M., Pirog, A., Jung, H. (2019). Cost of higher education: For-profit universities and online learning. The Social Science Journal, 1-9. https://doi.org/10.1016/j.soscij.2019.03.010

Hamidi, H., Chavoshi, A. (2018). Analysis of the essential factors for the adoption of mobile learning in higher education: A case study of students of the University of Technology. Telematics and Informatics, 35, 1053-1070. https://doi.org/10.1016/j.tele.2017.09.016

Hamidi, H., Jahanshaheefard, M. (2019). Essential factors for the application of education information system using mobile learning: A case study of students of the university of technology. Telematics and Informatics, 38, 207-224. https://doi.org/10.1016/j.tele.2018.10.002

Harfitta, G., Mei, J., Chowb, L. (2018). Transforming traditional models of initial teacher education through a mandatory experiential learning programme. Teaching and Teacher Education, 73, 120-129. https://doi.org/10.1016/j. tate.2018.03.021
Hernández, R. (2012). Does continuous assessment in higher education support student learning? Higher Education, 64(4), 489-502. https://doi.org/10.1007/s10734-012-9506-7

Hosseini, A. (2011). University student's evaluation of creative education in universities and their impact on their learning. Procedia - Social and Behavioral Sciences, 15, 1806-1812. https://doi.org/10.1016/j.sbspro.2011.04.007

Klement, M. (2015). How to Educate University Students: Analysis of Students' Attitudes to Forms, Methods, and Tools Applied within the Process of Education. Procedia Social and Behavioral Sciences, 203, 134-140. https://doi. org/10.1016/j.sbspro.2015.08.271

Klement, M., Chráska, M., Chrásková, M. (2015). The Use of the Semantic Differential Method in Identifying the Opininons Of University Students on Education Realized Through e-learning. Procedia - Social and Behavioral Sciences, 186, 1214-1223. https://doi.org/10.1016/j.sbspro.2015.04.165

Kovacova, M., Vackova, L. (2015). Cost of higher education: For-profit universities and online learning. The Social Science Journal, 1-8.

Leal Filho, Y., Raath, S., Lazzarini, B., Vargas, V., de Souza, L., Anholon, R., ... Orlovic, V. (2018). The role of transformation in learning and education for sustainability. Journal of Cleaner Production, 199, 286-295. https://doi.org/10.1016/j.jclepro.2018.07.017

Leaman, L., Flanagan, H. (2013). Authentic Role-playing as Situated Learning: Reframing teacher education methodology for higher-order thinking. Studying Teacher Education , 9, 4561. https://doi.org/10.1080/17425964.2013.77 $\underline{1573}$

Maclellan, E. (2004). How convincing is alternative assessment for use in higher education? Assessment and Evaluation in Higher Education, 3, 311-321. https://doi. org/10.1080/0260293042000188267

Manţa, Ş.G., Şarlea, M., Vaidean, V.L. (2015). Comparative Analysis of University Education 
Systems from the Central and Eastern European Countries. Procedia Economics and Finance, 1276-1288. https://doi.org/10.1016/ $\underline{\text { S2212-5671(15)01505-1 }}$

McDonald, M., Bowman, M., Brayko, K. (2013). Learning to see students: Opportunities to develop relational practices of teaching through community-based placements in teacher education. Teachers College Record, 115, 1-35.

Mingazova, M. (2014). Modification of the Active Learning Methods in Environmental Education in Russian Universities. Procedia - Social and Behavioral Sciences, 131, 85-89. https://doi. org/10.1016/j.sbspro.2014.04.083

Monereo, C.W., Alvarez, I. (2013). Changing university teacher's identity: Training based on dramatized incidents. Infancia y Aprendizaje, 36(3), 323-340. https://doi. org/10.1174/021037013807533043

Monereo, C.W., Weise, C., Alvarez, I. (2013). Cambiar la identidad docente en la universidad. Formación basada en incidents dramatizados. Journal for the Study of Education and Development, 36, 323-340. https://doi. org/10.1174/021037013807533043

Mork, O., Hansen, I., Strand, K., Giske, L., Kleppe, P. (2016). Manufacturing Education- Facilitating the Collaborative Learning Environment for Industry and University. Procedia CIRP, 54, 59-64. https://doi.org/10.1016/j. procir.2016.04.208

Muller, E., Tucker, M. (2017). Feedback on feedback practice: perceptions of students and academic. Assessment y Evaluation in Higher Education, 266-288. https://doi.org/10.1080/0 2602938.2015.1103365

Nicol, D., Macfarlane-Dick, D. (2006). Formative assessment and self-regulated learning: a model and seven principles of good feedback practice. Studies in Higher Education, 31, 199-218. https://doi.org/10.1080/03075070600572090

Postareffa, L., Virtanen, V., Katajavuoric, K., Lindblom-Ylännea, S. (2012). Academics' conceptions of assessment and their assessment practices. Studies in Educational Evaluation, 38, 84-92. https://doi.org/10.1016/j. stueduc.2012.06.003

Putnam, R., Borko, H. (2000). What do new views of knowledge and thinking have to say about research on teacher learning? Educational Researcher, 29(1), 4-15. https://doi. org/10.3102/0013189X029001004

Rieckmann, M. (2012). Future-oriented higher education: Which key competencies should be fostered through university teaching and learning? Futures, 44, 127-135. https://doi.org/10.1016/j.futures.2011.09.005

Ryan, M. (2011). Improving reflective writing in higher education: A social semiotic perspective. Teaching in Higher Education, 16, 99111. https://doi.org/10.1080/13562517.2010 .507311

Šolc, M., Legemza, J., Sütőová, A., Girmanová, L. (2012). Experiences with Utilizing e-learning in Education Process in University Environment. Procedia - Social and Behavioral Sciences, 46, 5201-5205. https://doi.org/10.1016/j. sbspro.2012.06.409

Tsimane, T., Downing, C. (2020). Transformative learning in nursing education: A concept analysis. International Journal of Nursing Sciences, 7(19), 91-98. https://doi.org/10.1016/j. ijnss.2019.12.006

Tvenge, N., Ogorodnyk, O. (2018). Development of evaluation tools for learning factories in manufacturing education. Procedia Manufacturing, 23, 32-38. https://doi.org/10.1016/j. promfg.2018.03.157

Vanclay, J.K. (1996). The future of forestry education. Institute of Foresters of Australia Newsletter, 37(2), 2-6.

Viberga, O., Hatakkab, M., Bältera, O., Mavroudia, A. (2018). The current landscape of learning analytics in higher education. Computers in Human Behavior, 89, 98-110. https://doi.org/10.1016/j.chb.2018.07.027

Warford, M. (2011). The zone of proximal teacher development. Teaching and Teacher 
Education, 27(2), 252-258. https://doi.org/10.1016/j.tate.2010.08.008

Willamo, R., Helenius, L., Holmström, C., Haapanen, L., Kolehmainen, L. (2018). Learning how to understand complexity and deal with sustainability challenges-A framework for a comprehensive approach and its application in university education. Ecological Modelling, 370, 1-13. https://doi.org/10.1016/j. ecolmodel.2017.12.011
Zeichner, R. (2010). Rethinking the connections between campus courses and field experiences in college-and university-based teacher education. Journal of Teacher Education, 61,89-99. https://doi.org/10.1177/0022487109347671

Zhang, Z., Cui, Q. (2018). Collaborative Learning in Higher Nursing Education: A Systematic Review. Journal of Professional Nursing, 35(5), 378-388. https://doi.org/10.1016/j. profnurs.2018.07.007 